\title{
AN EXPERIMENTAL ATTEMPT TO TRANSMIT PRIMARY ATYPICAL PNEUMONIA IN HUMAN VOLUNTEERS ${ }^{1}$
}

\author{
BY THE COMMISSION ON ACUTE RESPIRATORY DISEASES 2
}

\author{
(From the Respiratory Diseases Commission Laboratory, Regional Hospital, Section 2, Fort Bragg, \\ North Carolina)
}

(Received for publication July 17,1944 )

\section{INTRODUCTION}

During the past 10 years, the clinical and roentgenographic features of primary atypical pneumonia have been the subject of numerous reports $(1,2)$. Sufficient data have accumulated to establish this disease as a definite clinical entity, distinct from the usual forms of bacterial pneumonia. Etiological studies, conducted in many laboratories, have yielded divergent results, although in some instances filtrable agents have been isolated (3). Laboratory investigations to date have been restricted considerably because of the lack of a uniformly susceptible animal host. At the present time, identity of the infectious agent, the mode of transmission, and nature of communicability of the disease must be considered unknown.

The purpose of this communication is to report the results of a preliminary experiment conducted in human volunteers inoculated with respiratory tract secretions of patients ill with primary atypical pneumonia. The principal objective of the study was to obtain data concern-

1 This investigation was supported through the Commission on Acute Respiratory Diseases, Board for the Investigation and Control of Influenza and Other Epidemic Diseases in the Army, Preventive Medicine Service, Office of The Surgeon General, United States Army, and by grants from the Commonwealth Fund, the W. K. Kellogg Foundation, the John and Mary R. Markle Foundation, and the International Health Division of the Rockefeller Foundation to the Board for the Investigation and Control of Influenza and Other Epidemic Diseases for the Commission on Acute Respiratory Diseases.

2 Members of the Commission on Acute Respiratory Diseases are: J. H. Dingle, Major, M.C., A.U.S., Director; T. J. Abernethy, Major, M.C., A.U.S.; G. F. Badger, Captain, M.C., A.U.S.; J. W. Beard, M.D.; N. L. Cressy, Major, M.C., A.U.S.; A. E. Feller, M.D.; A. D. Langmuir, Captain, M.C., A.U.S.; C. H. Rammelkamp, M.D.; E. Strauss, Captain, M.C., A.U.S.; and Hugh Tatlock, 1st Lieutenant, M.C., A.U.S., National Research Council Fellow. ing the transmissibility of the disease in the natural host.

\section{METHODS AND MATERIALS}

Site of experiment. The study was conducted at a Civilian Public Service camp near Gatlinburg, Tennessee, in the Great Smoky National Park. A group of buildings, formerly used by the Civilian Conservation Corps, provided facilities for accommodating approximately 140 men. A small infirmary, apart from the main dormitories and normally equipped to care for 9 patients, was used to house the volunteers.

Subjects. All of the men were conscientious objectors who had volunteered for the experiment. From a group of 23 men, 15 were selected, after careful clinical, bacteriological, and roentgenographic study, as suitable for inoculation. An additional group of 16 volunteers was chosen for the purpose of observing the patterns of respiratory illness at the camp during the conduct of the experiment.

Isolation. The facilities of the infirmary prevented strict isolation of each individual in the inoculated group. Twelve men were quartered in double-decked bunks in one large room, 2 men shared a room of moderate size, and 1 individual occupied a single room. The members of the entire group were in close contact with each other at all times. They shared a common lavatory. The men were allowed restricted out-of-door recreational privileges, but had no immediate personal contact with the other campers nor were they allowed to enter other buildings. The attending physicians and assistants wore gowns and masks when in contact with subjects in the infirmary.

The non-inoculated group of 16 men pursued their normal duties in or around the camp, lived in the common dormitories, shared the common mess hall, and were in normal contact with their fellows, but were not allowed furloughs.

Observation of non-inoculated group. This group of 16 men was observed 3 times weekly for evidences of respiratory disease, at which time a history of the presence and severity of certain constitutional and localizing respiratory symptoms was made. Significant physical signs were recorded. Roentgenograms of the chest were taken weekly throughout the experiment. Throat cultures were taken on each man prior to and during the study. A differential and total leukocyte count was also made at the beginning of the study. Sera were obtained from each . man prior to and at the end of the experiment for the purpose of serological tests. 
TABLE I

Clinical and laboratory data on patients from whom specimens were obtained

\begin{tabular}{|c|c|c|c|c|c|c|}
\hline \multirow[b]{2}{*}{ Patient } & \multirow[b]{2}{*}{ Lobes involved } & \multicolumn{2}{|c|}{ Specimen } & \multicolumn{3}{|c|}{ Sera } \\
\hline & & Day of disease & Type* & $\begin{array}{c}\text { Day of } \\
\text { disease } \\
\text { obtained }\end{array}$ & $\underset{\text { titer }}{\text { Cold }}$ & $\begin{array}{l}\text { Strep. (Rocke- } \\
\text { feller No. 344) } \\
\text { agglutinin titer }\end{array}$ \\
\hline A. N. & L.L. & 4 & T.W. & $\begin{array}{r}5 \\
8 \\
16\end{array}$ & $\begin{array}{l}8 \\
8 \\
-\dagger\end{array}$ & $\begin{array}{l}10 \\
10 \\
40\end{array}$ \\
\hline M. F. & L.L. & 3 & $\begin{array}{l}\text { T.W. } \\
\text { Sp. }\end{array}$ & $\begin{array}{r}3 \\
10 \\
18\end{array}$ & $\begin{array}{r}16 \\
512 \\
-\end{array}$ & $\begin{array}{l}20 \\
10 \\
20\end{array}$ \\
\hline W. K. & $\begin{array}{l}\text { R.U., R.M., } \\
\text { R.L., L.L., }\end{array}$ & 3 & T.W. & $\begin{array}{r}3 \\
15 \\
20\end{array}$ & $\begin{array}{r}0 \\
512 \\
256\end{array}$ & $\begin{array}{l}20 \\
10 \\
20\end{array}$ \\
\hline L. R. & $\begin{array}{l}\text { R.U., R.M., } \\
\text { R.L. }\end{array}$ & 3 & T.W. & $\begin{array}{r}3 \\
17 \\
26\end{array}$ & $\begin{array}{r}0 \\
65,536 \\
4,096\end{array}$ & $\begin{array}{l}20 \\
40 \\
20\end{array}$ \\
\hline R. M. & R.L. & 3 & T.W. & $\begin{array}{l}3 \\
8\end{array}$ & - & $\begin{array}{l}20 \\
20\end{array}$ \\
\hline R. D. & R.L., L.L. & 13 & T.W. & $\begin{array}{l}12 \\
21 \\
32\end{array}$ & $\begin{array}{r}256 \\
4,096 \\
256\end{array}$ & $\begin{array}{l}20 \\
20 \\
20\end{array}$ \\
\hline R.S. & $\begin{array}{l}\text { L.U., L.L., } \\
\text { R.U., R.L. }\end{array}$ & 12 & $\begin{array}{l}\text { T.W. } \\
\text { Sp. }\end{array}$ & $\begin{array}{r}3 \\
12 \\
19\end{array}$ & $\begin{array}{c}0 \\
16,384 \\
4,096+\end{array}$ & $\begin{array}{r}10 \\
40 \\
160\end{array}$ \\
\hline
\end{tabular}

*T.W. = Throat Washings; Sp. = Sputum.

$\dagger-=$ Test not done.

Inocula. Throat washings and sputa were collected from 7 patients admitted to the wards of the Regional Hospital, Fort Bragg, during the previous 4 months (Table I). Each of these patients was ill with the characteristic syndrome of primary atypical pneumonia (lg), confirmed by roentgenogram. Five of the 7 patients developed "cold agglutinins" in their sera during the course of the disease. Specimens were collected from 4 patients on the third day of the disease; in the remaining 3 cases, collections were made on the fourth, twelf th, and thirteenth days, respectively, during which time the patients were severely ill. Immediately after collection, the specimens were placed in lusteroid tubes and frozen in a carbon dioxide ice-alcohol bath. They were stored at $-72^{\circ} \mathrm{C}$. until ready for use.

Roentgenograms. The standard U. S. Army portable Picker field $x$-ray unit, equipped with gasoline-driven generator, was employed in taking all chest films. All exposures were made with the subject in the upright position and at a distance of 72 inches for $3 / 4$ second, using intensifying screens. Processing of all exposed films was accomplished at the camp by the use of the standard U. S. Army Developing Unit.

Cold agglutinins. Tests to determine the presence of cold agglutinins were performed on the sera of all patients, both in the inoculated and non-inoculated groups (4). A modification of the technique originally advanced by Peterson, Ham, and Finland (5) was employed. The blood was allowed to clot at room temperature and the serum separated by centrifugation. Sera were then stored at $4^{\circ} \mathrm{C}$. until ready for use. Serial 2-fold dilutions of serum in physiological salt solution were made in $0.5 \mathrm{ml}$. volume in Wassermann tubes; the final dilution in the first tube was $1: 4$. One half $(0.5) \mathrm{ml}$. of 0.2 per cent washed Group 0 human cells (not over 24 hours old) was then added to each tube. The tubes were shaken vigorously and kept overnight at $4^{\circ} \mathrm{C}$. Readings were made immediately upon removal of the tubes from the icebox. Only positive tests which failed to persist after the tubes had stood at room temperature for 1 hour were accepted. Titers of $1: 32$ or higher were considered significant.

Agglutination tests. The sera of all individuals, both in the inoculated and non-inoculated groups, were tested for the presence of agglutinins to an indifferent streptococcus. The strains employed were Rockefeller No. $344,,^{2}$ as well as 19 individual strains isolated from throat washings and sputum obtained from both donors and recipients of the inoculum. The technique employed was that described by Thomas et al. (6).

This strain was kindly supplied by Dr. Frank L. Horsfall, Jr. 


\section{PRELIMINARY STUDIES}

Epidemiology. Prior to the inoculation of volunteers, a brief epidemiological survey of the camp was made. The purpose of this survey was to determine the incidence of respiratory illnesses during the 12 -month period preceding the initiation of the experiment, to secure evidence of the existence of primary atypical pneumonia at the camp, and to collect data regarding illnesses which had occurred among the men who volunteered for the experiment.

Tabulation of the monthly incidence of respiratory illnesses at the camp from October 1, 1942, to August 31, 1943 , and during the time the experiment was in progress, showed the expected seasonal variations. The majority of these illnesses were short in duration and mild in nature, and simulated the common cold. Only a small proportion required infirmary care, and then usually for less than 1 week. No definite evidence could be found that atypical pneumonia had existed at the camp prior to the initiation of the experiment, although in some individuals the disease may have been present and unrecognized for lack of roentgenographic study. The illness in one patient may have been significant; he had had a chronic cough for several weeks and a single roentgenogram was interpreted as showing "pneumonitis and questionable bronchiectasis."

Roentgenographic survey. As an adjunct to the above study, a roentgenographic survey was undertaken at the camp immediately prior to the experiment. Single films of the chest were taken on 123 individuals who were either members of the camp or closely identified with its administration. In no instance was an infiltrative lesion characteristic of atypical pneumonia encountered.

\section{EXPERIMENTAL STUDY}

Prior to inoculation, the 15 volunteers were segregated in the camp infirmary. Over a period of 5 days (October 3 to 8,1943$)$, each volunteer was examined daily and careful note was made of the development of any respiratory symptoms. The oral temperature and the pulse rate were recorded at 4-hour intervals, daily; the respiratory rate was taken twice, daily. During this period, a throat culture, bleeding, complete blood count, urinalysis, and roentgenogram were performed on each patient. Twelve individuals experienced no respiratory symptoms during this period of preliminary observation; 3 individuals developed respiratory illnesses as follows:

T. W. on October 4 noted headache, sneezing, slight soreness of nasopharynx, hoarseness, and dry cough. Physical examination was essentially negative. On October 6, coryza, nasal obstruction, and discharge were more evident. Symptoms of a mild respiratory illness without fever or significant physical signs continued for approximately 10 days. He was not inoculated but was not removed from the experimental group.

D. M. developed symptoms and signs of a mild afebrile respiratory infection 36 hours after entering the infirmary. The principal symptoms were those of coryza. No significant physical signs were apparent. By October 10, he was considerably improved and received 1 day's inoculations.

V. M. noted sneezing, dryness of the nasopharynx, and sore throat on October 6, 3 days after entry into the infirmary. Symptoms and signs of a mild infection of the upper nasal passages continued for 1 week. The patient remained afebrile throughout. He was not inoculated but was kept in the experimental group.

The illnesses of these 3 individuals complicated the experiment considerably. These infections were mild in nature, however, and similar in their manifestations to infections occurring simultaneously in other campers. None of the 3 men developed roentgenographic evidence of pulmonary infiltration at this time or subsequently.

Notwithstanding the development of these minor illnesses, the inoculations were given to the other 12 volunteers over a period of 3 days (October 8 to 10). Each patient received an inoculation of unfiltered throat washings (in meat-infusion broth or Ringer's solution) as well as a pool of unfiltered throat washings and sputa intranasally and by inhalation, 3 times daily, for 3 consecutive days. The intranasal inoculations were given with the aid of a standard nasal atomizer. The inhalation of inoculum was accomplished by the use of a nebulizer" which emitted a fine cloud of vapor. Patients were instructed to inhale deeply $\mathbf{5 0}$ times as the mouth of the nebulizer was held below the soft palate. With each inhalation, the fine vapor of inoculum could be seen being sucked downward toward the lower respiratory tract. Immediately following the separate inoculations, patients reclined in the supine position with the head extended slightly, for a period of 15 minutes. Each of the 12 patients received the full course of inoculations in an identical manner, and approximately $8 \mathrm{ml}$. were given to each man. One additional volunteer (D. M.) was given 1 day's inoculation, totaling $2.5 \mathrm{ml}$.

During the post-inoculation period of 5 weeks, the volunteers were observed daily for the development of symptoms and physical signs of respiratory disease. Observations of the temperature, and of the pulse and respiratory rates, were continued. Roentgenograms of the chest were taken twice weekly, or of tener if indicated. Leukocyte counts with Schilling differential indices were performed twice weekly. Blood for serological study was obtained at weekly intervals. Throat cultures were taken weekly. Patients in need of complete bed rest and nursing care were transported to a nearby hospital.

\section{RESULTS IN NON-INOCULATED GROUP}

Clinical findings. Respiratory illnesses of mild severity and short duration developed in 13 of the 16 individuals who were permitted to pursue their normal duties about the camp but who received no inoculation. The time of development of respiratory disease in this group is shown in Figure 1. Three patients pre-

\footnotetext{
- Made by Vaponefrin Company.
} 


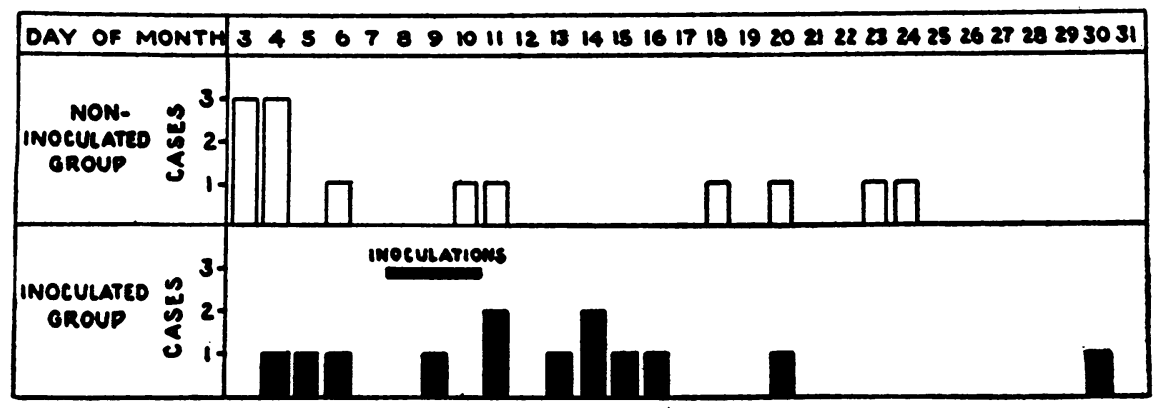

Fig. 1. Day of Onset of Respiratory Disease in InOculated AND Non-INoculated Groups

Note: Three patients in non-inoculated group presenting evidence of respiratory infection on October 3 developed symptoms on September 24, October 1 and 3, respectively.

sented evidence of mild upper respiratory infections when first examined on October 3. These illnesses began on September 24, October 1, and 3 , respectively. Three additional patients developed symptoms on October 4 . During the succeeding 5 weeks, respiratory disease developed in the remainder of the group in sporadic fashion. Constitutional symptoms (chilliness, malaise, or headache) during the course of their infection were reported by only 3 individuals (Figure 2). Localizing symptoms, e.g., coryza, sore throat, and occasionally cough and hoarseness, were the predominant features of these illnesses (Figure 3). Roentgenograms of the lungs were consistently negative in 15 individuals. In only 1 case was there suggestive evidence of pulmonary infiltration accompanying the respiratory infection. The clinical history on this patient's illness follows.

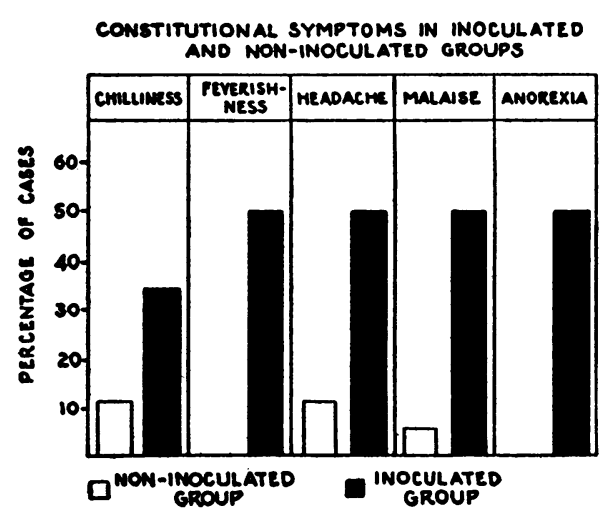

Fig. 2. Constitutional Symptoms in Inoculated and Non-Inoculated Groups
On October 3, K. B. inhaled a considerable amount of smoke while engaged in fighting a forest fire. That evening he noted a slight sore throat. The following day he complained of headache, sneezing, coryza, hoarseness, and cough. He reported to the dispensary, where his temperature was found to be $99.4^{\circ} \mathrm{F}$. and he was placed in quarters. On October 5 , his temperature was normal but the symptoms continued. On October 6, his symptoms were unchanged and the physical examination revealed tender lymph nodes, nasal obstruction and discharge, a small patch of exudate on the pharynx, and scattered rhonchi over the lungs. Roentgenograms of the lungs taken on September 29, before the development of this infection, and on October 6, showed no pulmonary lesion. By October 8 , he was sufficiently improved to be discharged from the infirmary, but cough and nasal symptoms continued. Physical examination at this time disclosed only tender cervical lymph nodes; no pharyngeal exudate was visible and the lungs were clear. During the next 10 days, while on duty, he was aware of intermittent headache, malaise, nasal discharge, sore throat, and cough, but nothing of significance was found on physical examination. A roentgenogram of the chest on October 14

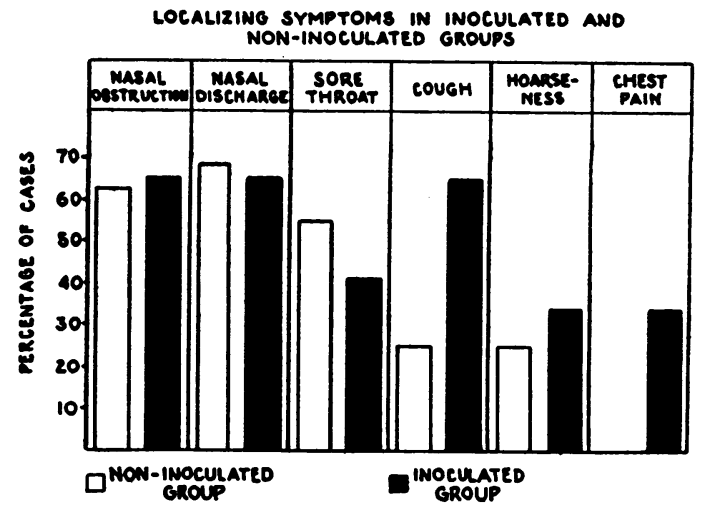

Fig. 3. Localizing Symptoms in Inoculated AND Non-Inoculated Groups 
was again negative. He was readmitted to the infirmary on October 18 because of exacerbation of all symptoms the previous night. He complained of cough and a dull frontal headache, and there was tenderness on pressure over the frontal sinuses, together with a purulent postnasal discharge. His temperature remained normal. On October 21, a roentgenogram of the chest was taken, which has since been interpreted as showing a questionable area of increased density of the mesial portion of the left lung. Opinion of the roentgenologists is divided as to whether this lesion represents peribronchial pneumonia or increased bronchovascular markings. The patient was able to resume his duties on October 27. A roentgenogram of the chest on November 2 was negative. No fine râles over the chest were detected at any time. The clinical diagnosis was sinusitis, pharyngitis, and bronchitis. One specimen of serum (November 5) was tested for the presence of cold agglutinins but none was detected.

Laboratory findings. Throat cultures from the non-inoculated group, prior to and during the course of the experiment, showed no significant deviation from the normal flora. One individual with a mild respiratory infection was shown to have a moderate growth of $\beta$-hemolytic streptococcus on a single culture. There was no evidence that this organism had spread to other individuals in the group. Leukocyte and differential counts on this group were normal.

Tests for the presence of cold agglutinins and agglutinins for an indifferent streptococcus (Rockefeller No. 344) were performed on speci- mens of sera taken prior to the experiment and 1 month later. Cold agglutinins were not present in significant titer $(1: 32)$ and there was no rise in titer of agglutinins for the streptococcus.

\section{RESULTS IN INOCULATED GROUP}

Respiratory illnesses developed in 10 of the 12 volunteers receiving the full 3 days' course of inoculations. In general, these illnesses were more severe and differed sharply from the infections observed in the non-inoculated group. The important data obtained following inoculation are given in Table II and in summary form below.

Day of onset of respiratory disease. Figure 1 shows the time of appearance of any symptom marking the onset of respiratory disease. In 3 individuals, respiratory disease began either during or immediately following the inoculations and was manifested by symptoms and/or signs localized to the nose or throat. Five individuals noted respiratory symptoms beginning from 5 to 8 days (October 13 to 16) following the first inoculation; one man noted symptoms on the twelfth day (October 20) and one noted symptoms on the twenty-second day (October 30). The development of the majority of these illnesses over a period of approximately 1 week

TABLE II

Clinical and laboratory data on patients in experimental group

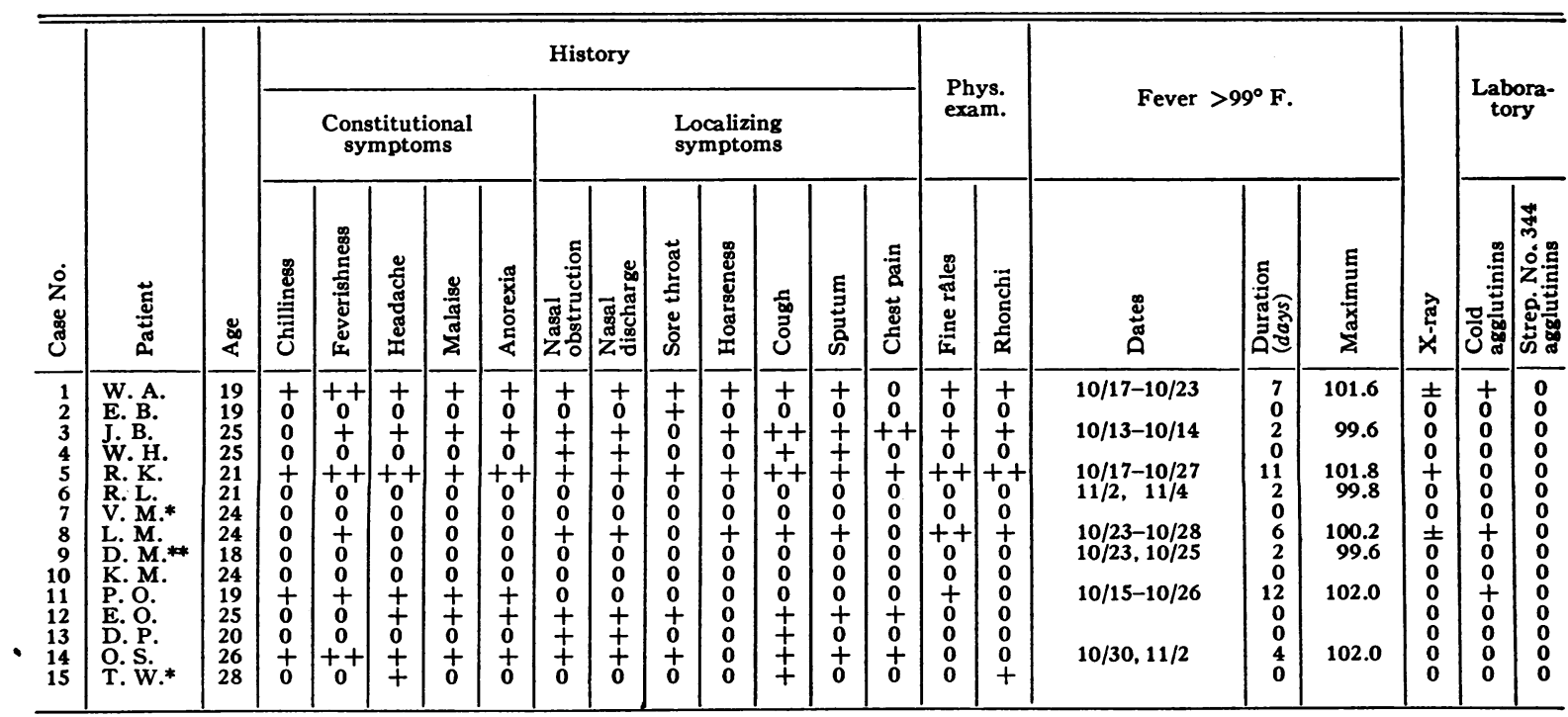

* Not inoculated.

** Received 1 day's inoculations October 10, 1943. 


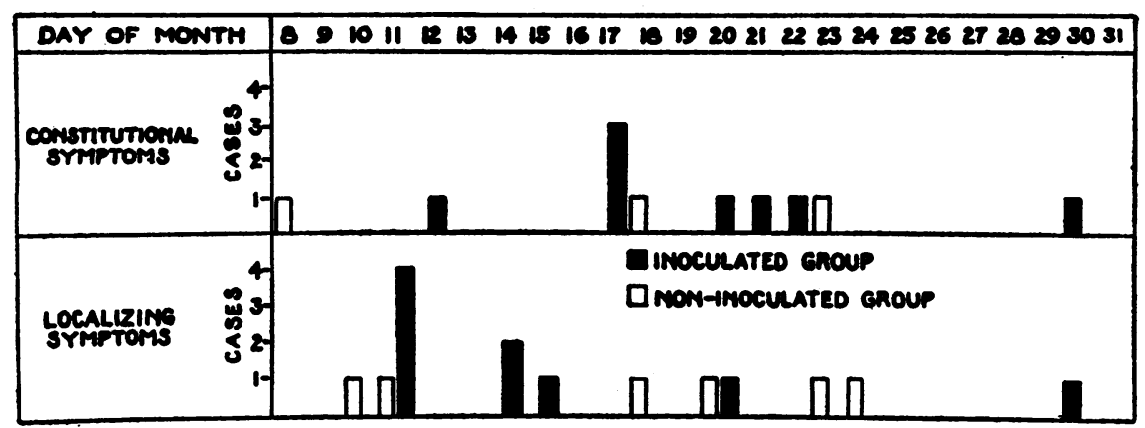

Fig. 4. Day of Onset of any Constitutional or Localizing Symptom from Date of Inoculation in InOculated and Non-Inoculated Groups

following the first inoculation is in sharp contrast to that observed in the non-inoculated group.

Constitutional symptoms. Seven patients developed significant constitutional symptoms which were generally of moderate severity, although in 3 patients (W. A., R. K., and O. S.), some of the symptoms were present in severe form (Table II). One individual (L. M.) presented only a single constitutional symptom. Chilliness was noted by 4 patients (Figure 2). In no instance was a frank rigor observed. Subjective sensation of feverishness was present in 6 patients. Dull frontal headache, malaise, and anorexia were each present in 6 cases. The time of development of constitutional symptoms differed somewhat among the 12 inoculated individuals (Figure 4). Three men noted onset of symptoms on October 17, 9 days following the first inoculation.

Localizing symptoms. Symptoms, localized either in the upper or lower respiratory tract, were present in 9 patients (Table II, Figure 3). Nasal obstruction and discharge were present in each of 8 patients. Sore throat, usually of mild severity, was noted by 5 individuals. Hoarseness was noted in one-third of the patients. Cough, usually of a dry paroxysmal character in the early stages, was observed in 8 patients. In 2 of these, the cough was severe, and in 7, it became productive. The sputa were invariably mucoid or mucopurulent and in no instance contained fresh or altered blood. Chest discomfort consisting of substernal "soreness" or "rawness" was observed by one-third of the patients. None of the patients developed a pleural type of pain. The day of onset of these localizing symptoms is shown in Figure 4.

Physical examination. Characteristic "sticky" subcrepitant râles (lg) were detected in the lungs of 5 patients. In 2 , the râles were persistent and continuously heard over localized areas. In the remaining 3 patients, the râles were localized to given areas but were transitory in nature. Rhonchi were audible in 4 patients. Abnormal signs in the nose and throat were detected in 8 patients having symptoms from these regions; these signs, however, were usually minimal. Two patients developed purulent discharge from the nose. Moderate injection of the throat usually accompanied symptoms of sore throat. The remainder of the physical examination showed no abnormalities.

Fever. Eight patients developed fever $\left(99^{\circ} \mathrm{F}\right.$. or above) following the inoculations (Table II). The temperature charts of these patients (Figure 5) show variability not only in the time of development (October 13 to November 4) but also in the height of the temperature response $\left(99.2\right.$ to $102^{\circ} \mathrm{F}$.). There were 5 individuals (Cases 11, 1, 5, 8, and 14) who showed maximum temperatures of $100^{\circ} \mathrm{F}$. or above and who had associated symptoms of respiratory illness. The development of significant fever was first observed in these individuals in a period varying between 7 and 22 days after the first inoculation. Three of these 5 men showed febrile responses beginning 7 to 9 days after the first inoculation. There were 3 volunteers who developed slightly . abnormal temperatures of 1 to 2 days' duration (Cases 1, 9, and 6). Only 1 individual (Case 3) 


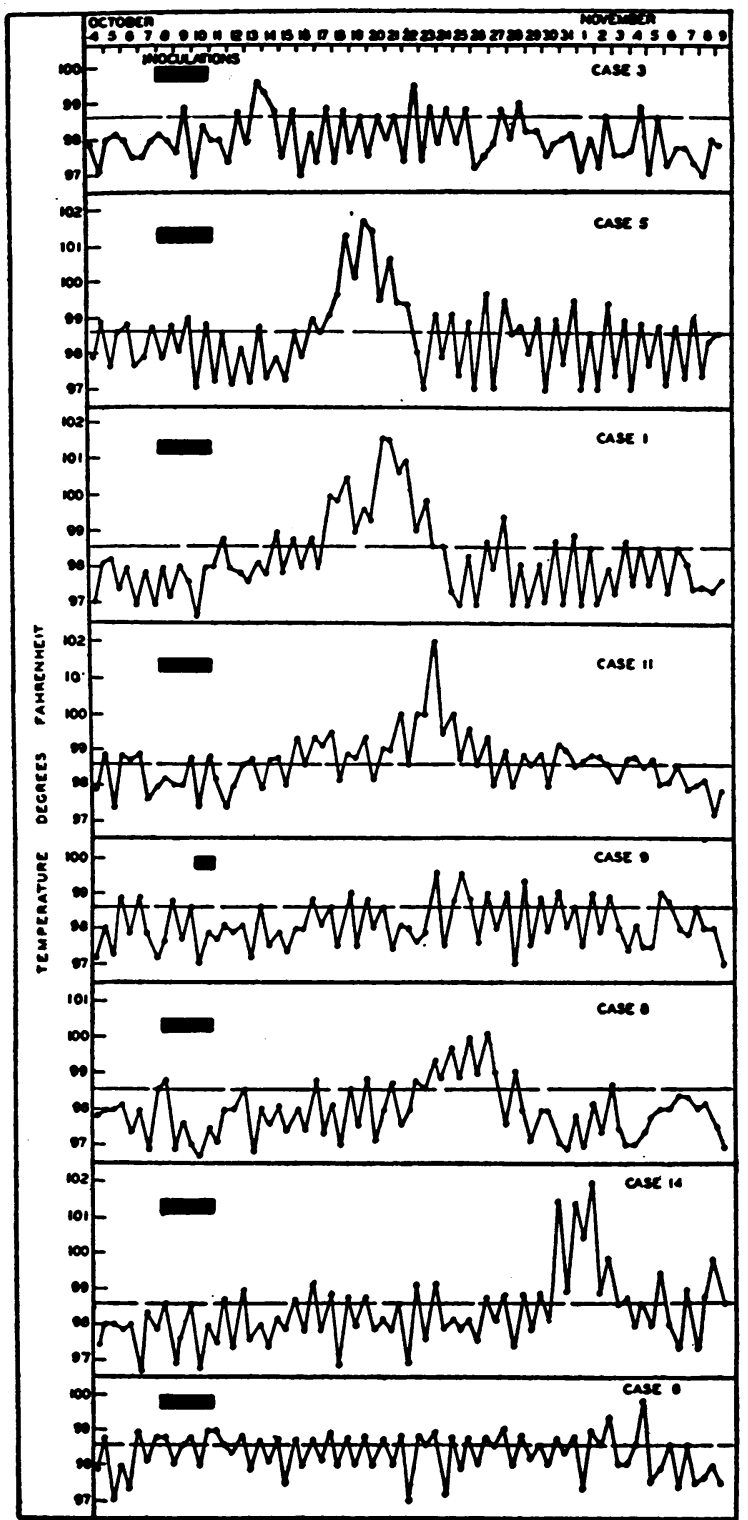

Fig. 5. Composite Temperature Charts of 8 Volunteers Who Developed Fever ( $99^{\circ} \mathrm{F}$. Or Above) Following THE InOculations

in the latter group manifested systemic symptoms of illness.

Roentgenograms. On review of all films without knowledge of the clinical history, 1 patient (R. K., Case 5), in the opinion of the roentgenologists, showed evidence of a patchy infiltration of both lung bases, more marked on the left. This patient had the characteristic symptomatology and physical signs usually accompanying primary atypical pneumonia. The films of 2 other pa- tients (W. A., Case 1, and L. M., Case 8) showed similar findings in single lobes, but to a less marked degree. Patients R. K. and W. A. were sufficiently ill to be hospitalized. In these instances, however, the pulmonary infiltrations were mild in degree and not so extensive as those usually observed in atypical pneumonia as seen in a hospital ward.

Laboratory findings. Cold agglutinins were detected in the sera of 3 patients (Table II). One patient (L. M., Case 8), who developed a febrile illness of 6 days' duration with accompanying symptoms and signs of respiratory disease, had a cold agglutinin titer of $1: 128$ on October 28 and 1:256 on October 31. Another patient (P. O., Case 11) developed cold agglutinins in titer of 1:64 following a febrile illness of 12 days' duration. A third patient (W. A., Case 1) showed a cold agglutinin titer of 1:128. Tests on the sera of the remaining patients were negative.

Agglutination tests with 20 strains of indifferent streptococci (Rockefeller No. 344, and 19 additional strains) showed no definite rises in antibody titers in the sera of any of the patients receiving inoculations.

Tests for the development of antibodies against influenza viruses A (PR8) and B (Lee) were done on samples of sera collected before and 3 weeks following the inoculations, employing the chicken red blood cell agglutination inhibition test of Hirst (7). No evidence of an increase in antibody titers to either virus was demonstrable in any of the specimens of convalescent serum.

With two exceptions, the leukocyte and Schilling differential counts in these patients showed no significant deviation from normal throughout the experimental period, despite the development of fever and/or severe respiratory symptoms. Two patients who were severely ill and required hospitalization developed a leukocytosis of 12,000 with an absolute increase in the total number of polymorphonuclear neutrophiles during the febrile period.

Results of weekly throat cultures showed no significant changes in bacterial flora immediately following the inoculations. The types of organisms present were those usually encountered in routine cultures from normal individuals with- 
out respiratory disease. $\alpha$-streptococci, $H$. influenzae, and gram-negative cocci (not further identified) were consistently predominant organisms throughout the experimental period. Beginning on October 20, however, $\beta$-hemolytic streptococci were recovered from 1 patient. On October 26 and again on October 29, similar organisms in moderate numbers were isolated from 4 other cases. On November 3, only 1 patient was found to be still harboring these organisms. There was no evidence that the presence of $\beta$-hemolytic streptococci was associated with a clinical illness in any of these patients.

\section{Case Reports}

Case 5, Figure 6. R. K., aged 21, had enjoyed good general health immediately prior to the experiment. His past history was marked by a severe attack of tonsillitis in 1936. He usually experienced one or two "head colds" a year, lasting approximately 7 days. The most recent infection occurred in February 1943. During the preinoculation period, no significant respiratory symptoms or signs were observed.

Mild nasal obstruction and discharge developed concurrently with the inoculations. On October 11, a dry cough developed which increased in severity so that by October 15 it was keeping the patient awake at night.
On October 17, the patient complained of chilliness, dull supra-orbital headache, and substernal discomfort following each cough. He did not appear ill at this time and the physical examination was essentially negative. The following day the patient appeared definitely ill for the first time. Constitutional symptoms of chilliness, feverishness, headache, malaise, and anorexia were conspicuously present and the temperature rose to $101.6^{\circ} \mathrm{F}$. Physical examination disclosed only questionable dullness over the right hilus posteriorly. On October 19, the patient's fever continued and all symptoms became aggravated. Examination revealed numerous fine and coarse moist râles at both bases, without other signs of infiltration. On this date, he was transferred to a hospital for further care, where he stayed for approximately 3 weeks. A roentgenogram of the chest on October 20 showed evidence of a bilateral infiltrative lesion consistent with that of primary atypical pneumonia. These findings were observed on successive daily films taken over the course of the next week, but with gradual clearing of the pneumonic process during this time. The febrile course was of 11 days' duration. Constitutional symptoms persisted until November 2. Fine râles were continuously heard over a period of approximately 1 week. Except for a cough lasting until November 7 , the patient made an uneventful recovery.

Comment. An acute febrile illness of moderate severity developed 9 days after the first inoculation. Symptoms and signs suggestive of

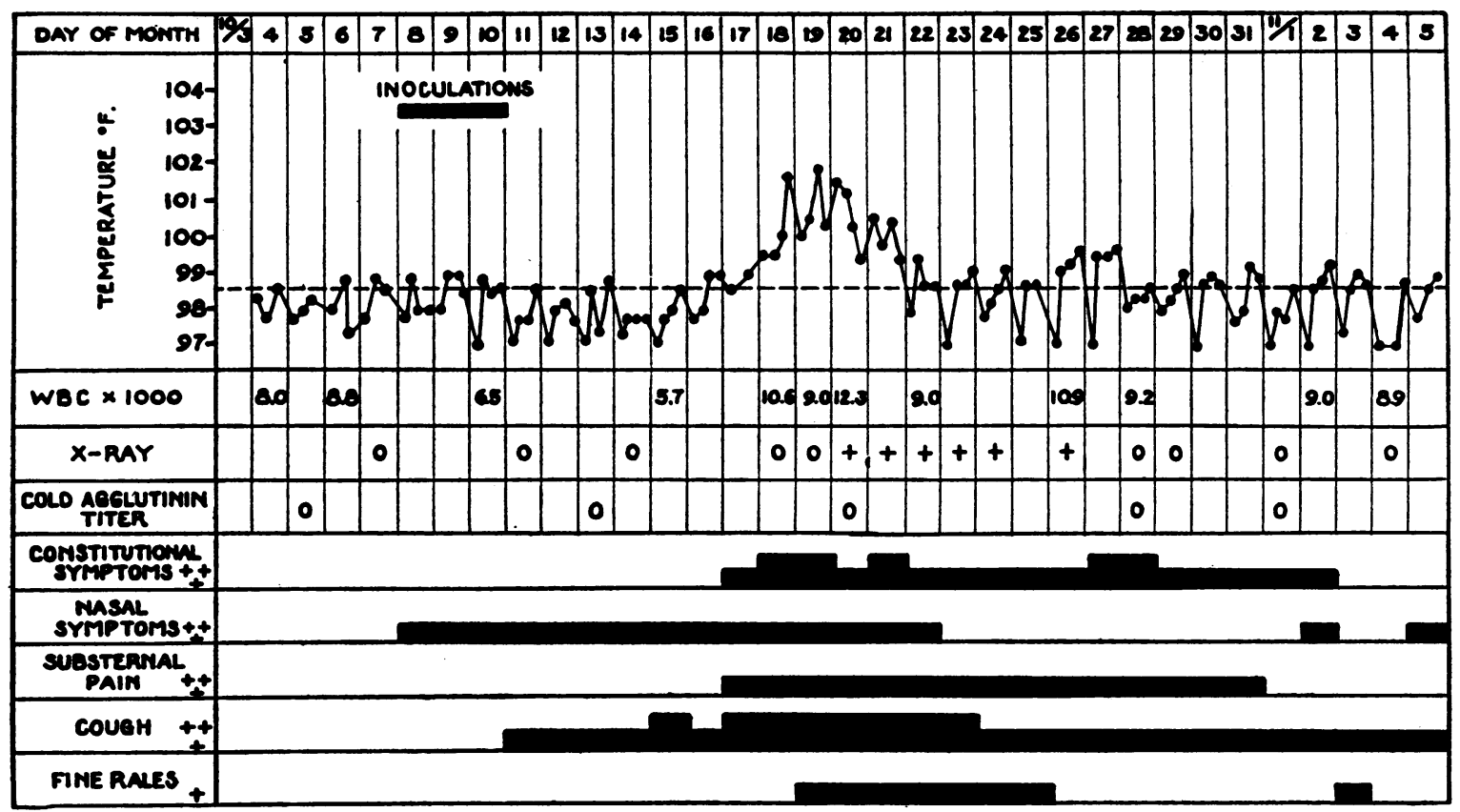

Fig. 6, Case 5. Clinical Chart of Patient Who Developed an Acute Febrile Respiratory Illness Resembling Primary Atypical Pneumonia

Note: $+=$ symptom or sign present and of mild degree; $++=$ symptom or sign present and of moderate severity. 


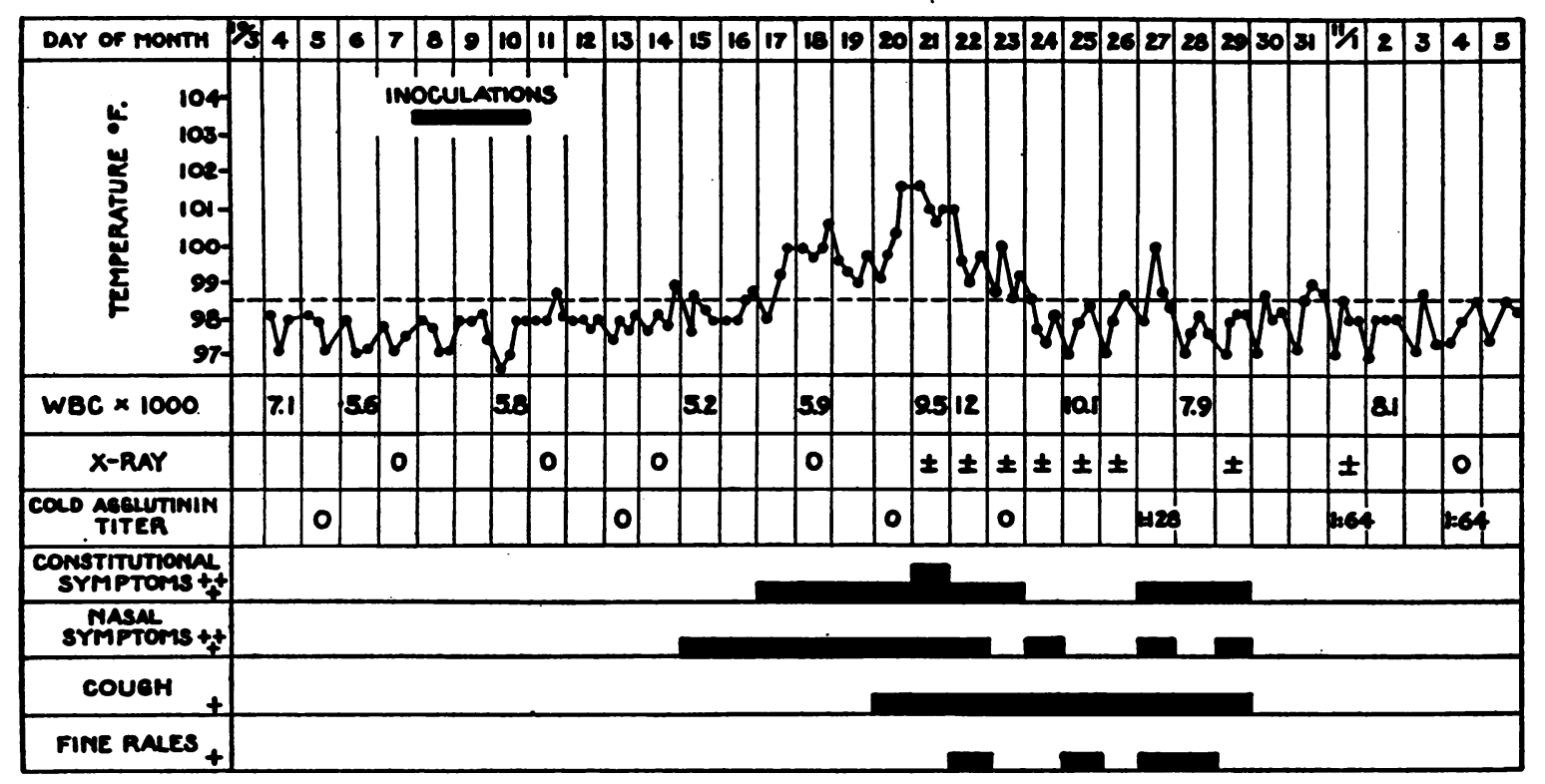

Fig. 7, Case 1. Clinical Chart of Patient Who Developed an Acute Febrile Respiratory Illness Resembling Primary Atypical Pneumonia

For explanation of symbols, see Fig. 6.

primary atypical pneumonia were noted. Evidence of a bilateral infiltrative lesion was obtained by roentgenograms of the chest. Convalescence was prolonged but the patient made an uneventful recovery.

Case 1, Figure 7. W. A., a 19-year-old camper, had always enjoyed good health except for minor head colds of short duration, occurring 3 or 4 times a year, and for a "streptococcus throat infection" in 1938, following which tonsillectomy was performed. Examination prior to the experiment disclosed no abnormal respiratory symptoms or signs except for marked deviation of the nasal septum.

No symptoms developed during the course of the inoculations. On October 14, the patient complained of slight sore throat and hoarseness; however, the physical examination was negative. On October 15, coryza and nasal obstruction were noted. Two days later, constitutional symptoms developed, consisting principally of headache, chilliness, malaise, anorexia, and fatigability. The temperature, which had previously been normal, rose to $100^{\circ} \mathrm{F}$. The patient looked mildly ill. All symptoms became aggravated the following day, and fever varied between $99.8^{\circ}$ and $100.6^{\circ} \mathrm{F}$. His condition was somewhat improved on October 19 and the temperature was lower. Constitutional symptoms continued, and he was still mildly ill. Physical examination disclosed no significant abnormalities of the respiratory tract. With the onset of cough and severe constitutional symptoms on October 20, the patient appeared more ill. There was questionable dulness to the percussion note at the right base, but no râles. He was transferred to a hospital on October 21. On this date, the lungs were negative to physical examination, but the roentgenogram revealed exaggerated markings in the mid-lung field adjacent to the right heart border. This abnormality gradually cleared over the next 10 days. Opinion of roentgenologists was divided as to the significance of the changes observed. Examination of the chest at daily intervals failed to reveal fine "sticky" râles over the area shown roentgenographically to be abnormal. A few fine râles, however, were audible at intervals over the left hilus. The temperature fell to normal by lysis on October 24 and thereafter remained normal. Convalescence was slow and there were no complications. Tests for the development of cold agglutinins in the serum were done subsequent to recovery. These showed a titer of $1: 128$ on October 27, with a progressive fall in titer in the samples taken on November 1, 4, and 12 .

Comment. The illness in this patient was of moderate severity. Constitutional symptoms, which marked the onset of the acute febrile course, developed 9 days after the first inoculation. The symptoms were characteristic of those usually associated with atypical pneumonia. Neither physical signs nor definite roentgenographic evidence of the disease, however, developed with any degree of certainty. The course of the disease was consistent with that of a moderately severe case of atypical pneumonia. 
Case 11, Figure 8. P. O., aged 19, gave a history of repeated infections of the respiratory tract during the past few years. These consisted principally of a head cold and sore throat which occurred approximately 6 times a year, lasting less than a week at a time. He had pneumonia in 1931 and 1936, and sinusitis and mastoiditis in 1934. During the previous 3 months, he had been free of respiratory infections.

The patient remained well during and immediately following the inoculations. Beginning on October 15 and continuing for approximately a week, there was slight afternoon or evening elevation of temperature to $99.4^{\circ}$ or $99.6^{\circ} \mathrm{F}$. There were no accompanying complaints and the patient was normally active. On the 21 st and 22nd of October, the temperature rose to $100^{\circ} \mathrm{F}$. At this time, he noted feverishness, chilliness, and frontal headache, but no abnormal physical findings were observed. He became mildly ill on October 23 with the development of fever $\left(102^{\circ} \mathrm{F}\right.$.), chilliness, feverishness, malaise, anorexia, sneezing, and slight nasal discharge. Except for moderate injection and hypertrophy of the pharyngeal lymph follicles, no significant physical signs were noted. The patient stated that his symptoms were more severe than those usually experienced with respiratory infections. On October 24, the temperature was lower and constitutional symptoms had abated materially. The abnormalities previously noted in the throat were still present. The lungs were clear by auscultation. The temperature fell by lysis and there was corresponding symptomatic improvement. On only one occasion, October 31 , were a few fine râles detected over the right axilla which persisted af ter coughing. Roentgenograms taken repeatedly, during and subsequent to the febrile course, failed to reveal evidence of pulmonary infiltration at any time. The patient made an uneventful convalescence.
Tests for the development of cold agglutinins were performed on the patient's sera, collected at weekly intervals throughout the period of observation. Agglutinins in low but significant titer $(1: 64)$ were detected in the serum sample of October 28.

Comment. This patient's illness consisted of an initial asymptomatic period, characterized by low-grade fever beginning 7 days after the first inoculation, which was followed by a sharp febrile symptomatic period of 1 week's duration. The symptoms were of moderate severity. Râles in the lungs were heard on only one occasion, late in the course of the disease. The roentgenograms did not show abnormal pulmonary shadows. Sera of the patient revealed the presence of cold agglutinins in significant titer. Except for the negative roentgenograms and the paucity of confirmatory lung signs, the patient developed a mild respiratory infection consistent with the diagnosis of atypical pneumonia.

Case 8, Figure 9. L. M., 24 years old, had always enjoyed good health. He usually experienced 2 head colds each winter, which lasted 2 or 3 days and were uncomplicated. His most recent respiratory infection occurred in January 1943.

No significant symptoms or signs developed during the pre-inoculation period or immediately following the inoculations. The patient was well until 12 days after the first inoculation, when he noted dryness of nose and pharynx, hoarseness, and a "tickling" cough, symptoms

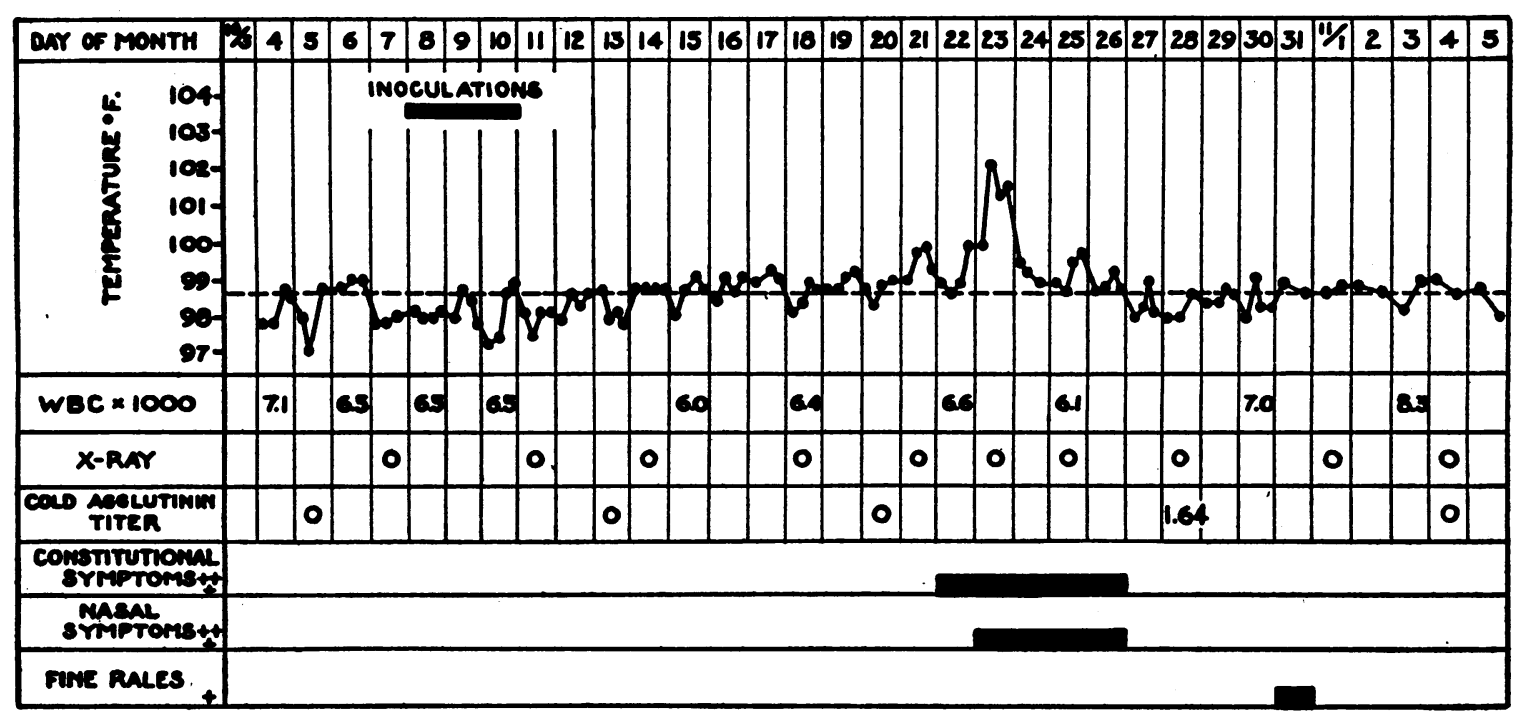

Fig. 8, Case 11. Clinical Chart of Patient Who Developed a Mild Febrile Respiratory ILlNess Following InOCULATION

For explanation of symbols, see Fig. 6. 


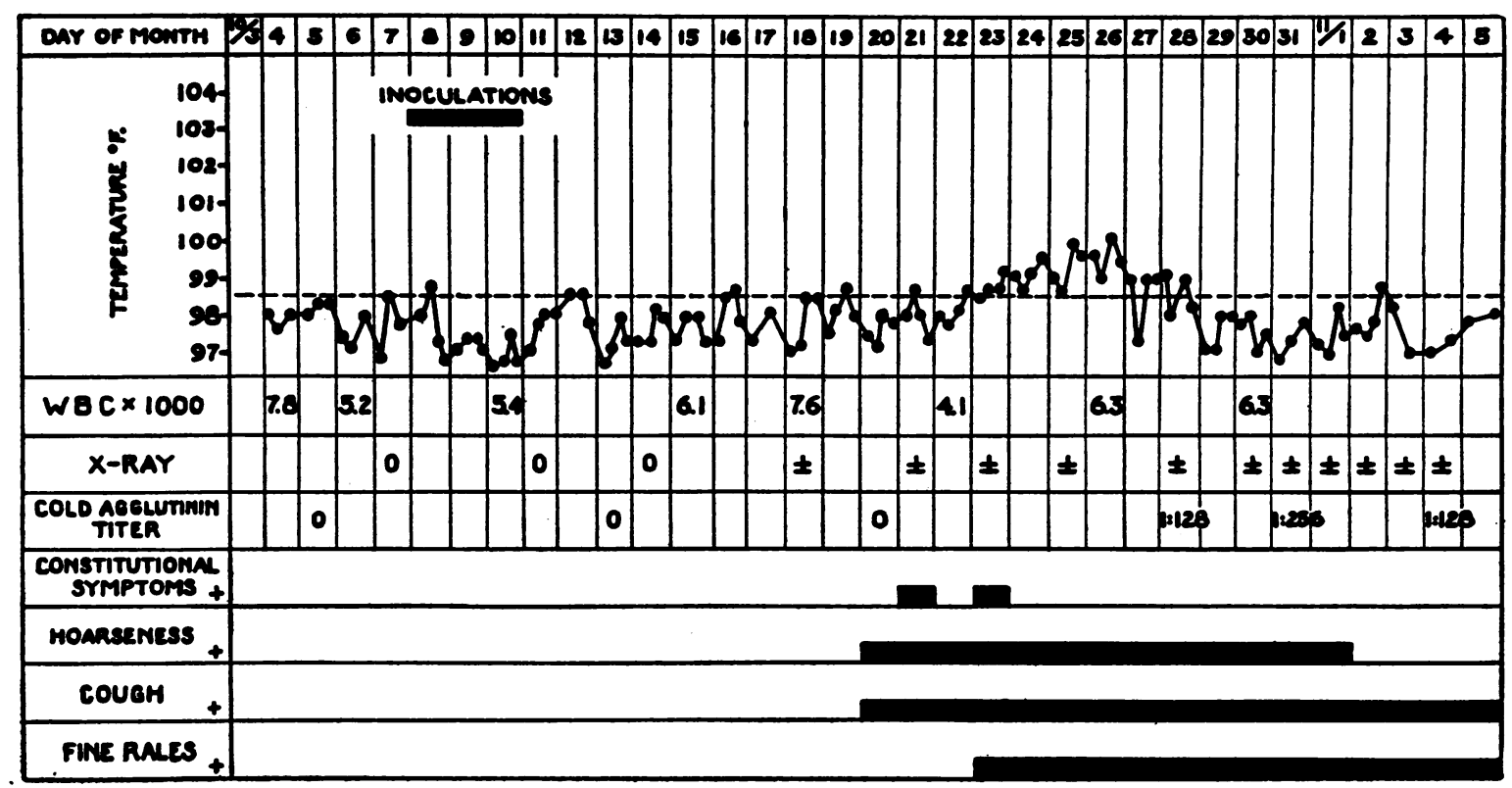

Fig. 9, Case 8. Clinical Chart of Patient Who Developed a Mild Respiratory Illness Resembling Primary Atypical Pneumonia

For explanation of symbols, see Fig. 6.

which were unlike those usually experienced with a cold. On October 21, there was slight nasal obstruction, injection of the pharynx, exudate on a portion of the pharyngeal lymphoid tissue, and aggravation of the cough. His symptoms continued, with dry cough and hoarseness as the most prominent features. On October 23, he noted slight feverishness, and fine inspiratory râles and rhonchi were detected for the first time over the lung bases. Slight evening elevation of temperature was noted on this date, and a low-grade fever continued during the next 5 days. The patient was less active than usual but not sufficiently ill to require bed rest. Râles and rhonchi persisted and were heard daily over both lung bases and over the right middle lobe. Rhonchi were no longer heard after November 2; a few râles were audible until November 11, a period of approximately 3 weeks. Cough persisted for about 4 weeks and eventually cleared. There were no complications.

Roentgenograms of the chest were consistently negative until October 18. On this date, the film showed increased bronchovascular markings at the left base. One roentgenologist who reviewed the films believed the infiltration was of sufficient degree to represent definite peribronchial pneumonia. The films taken on October 21 and 23 showed no change. On October 25, the increased markings were less evident. Residual changes were visible in frequent films taken over the course of the next 2 weeks. A final film on November 7 still showed a residual amount of increased markings at the left base.

Cold agglutinins developed in the sera of this patient with the cessation of the febrile period. A sample taken on October 28 gave a titer in dilution of $1: 128$. Subse- quent samples taken on October 31 and November 4 showed titers of $1: 256$ and $1: 128$, respectively.

Comment. This patient's illness was marked by an insidious onset, minimal constitutional symptoms, a short febrile course, protracted cough and lung signs, and questionable roentgenographic evidence of pulmonary infiltration. The illness was extremely mild and not incapacitating. Cough and hoarseness were the predominant symptoms. The development of cold agglutinins, together with the auscultatory findings, suggested the diagnosis of mild atypical pneumonia.

\section{DISCUSSION}

In this study, an attempt has been made to transmit primary atypical pneumonia in human volunteers. At the beginning of the experiment, it seemed of importance to determine whether or not atypical pneumonia existed at the camp, either in the immediate or in the remote past. For this purpose, a survey was conducted which showed a normal seasonal prevalence of respiratory disease in the 12 -month period preceding the initiation of the experiment. The data available from the dispensary records indi- 
cated that definite cases of pneumonia probably had not occurred. In addition, a roentgenographic survey, consisting of single films in 123 individuals, revealed no instance of atypical pneumonia. There was thus an indication that the disease under investigation was not then present in recognizable endemic form at the camp and that infections, if they could be produced successfully, might reasonably have resulted from the inoculations.

The observations made in this preliminary experiment indicate that respiratory disease of variable clinical patterns developed in a considerable proportion of human volunteers following inoculation with unfiltered secretions obtained from the respiratory tracts of patients with primary atypical pneumonia. The clinical course of illness in 3 individuals (Cases 1, 5, and 8) was similar to, if not identical with, that usually observed in patients with primary atypical pneumonia (lg). These 3 individuals showed suggestive roentgenographic evidence of pneumonia and 2 patients were sufficiently ill to be hospitalized. In another individual (Case 11), an illness resulted which was indistinguishable from atypical pneumonia except for the absence of roentgenographic evidence of the disease. This type of illness has previously been termed "bronchitis resembling atypical pneumonia" (lg). Six additional patients (Cases $2,3,4,12,13$, and 14, Table II) developed mild acute illnesses of the respiratory tract without pulmonary lesions demonstrable either by physical examination or by roentgenogram. Thus, there is a limited amount of experimental evidence from this preliminary study to indicate a possible relationship between atypical pneumonia and certain mild illnesses of the respiratory tract without accompanying pulmonary lesions. Others $(8,9 a$ and $b)$ have previously noted this relationship. Clinical and epidemiological evidence obtained from the study of atypical pneumonia at Camp Claiborne, La. (lg), and more recently at Fort Bragg (10a and b), also has supported this concept.

The course of illness which developed in these volunteers bore little resemblance to that usually observed either in individuals with the "common cold" or in subjects inoculated with filtered nasal secretions from patients with early acute "colds." Although symptoms and signs of inflammation of the nose and throat were noted in approximately two-thirds of the volunteers, it seemed significant that these manifestations were mild and often accompanied by definite constitutional symptoms and fever. Furthermore, two other groups of workers $(11,12)$ have shown that in human transmission experiments the incubation period of colds is generally short, usually 24 hours or less and probably not exceeding 4 days. Evidence from this experimental study indicates an incubation period of the acute illness in excess of 4 days. In view of the fact that unfiltered inoculum was used, however, and that mild respiratory disease was present in 3 volunteers at the beginning of the experiment, the possibilities exist that these infections may represent either bacterial complications of the "common cold" or instances of undifferentiated respiratory disease. These possibilities cannot be excluded with certainty, although the clinical course of disease in the inoculated patients did not support either concept.

The nature of illness developing in these volunteers likewise bore little resemblance to the classical picture of influenza, either experimentally or naturally acquired (13). Two other investigators $(14,15)$ have shown that an acute illness of abrupt onset usually follows within 24 to 48 hours after the experimental inoculation of influenza virus A into human volunteers. The subjects in this study developed their illnesses more than 48 hours after inoculation, and neither the clinical characteristics nor course of disease resembled typical influenza. Furthermore, none of the patients nor the men in the non-inoculated group showed serological evidence of an antibody rise to influenza viruses A or B during the period of the experiment.

The lack of more definite evidence of pulmonary infiltration in any of the roentgenograms was an unexpected finding, in consideration of the severity of illness manifested by some patients. The soft, patchy lesion with greatest density near the hilus, which is observed so frequently in atypical pneumonia, was not encountered. The maximum changes observed consisted of greatly exaggerated bronchovascular markings, which were seen to develop gradually at the base of one or more lobes and to undergo 
slow resolution over a period varying from 1 to 2 weeks. These findings, although minimal, were considered significant, and may be of importance to the concept of the possible relationship of certain forms of bronchitis to atypical pneumonia (lg).

In view of the serological reactions with an indifferent streptococcus (Rockefeller No. 344) obtained in certain cases of atypical pneumonia (6), a study was made of the relationship of this bacterium and other strains of indifferent streptococci to the respiratory illnesses occurring not only in the donors, but also in the recipients of the inoculum, i.e., the volunteers. Nine strains of indifferent streptococci were isolated from the inocula used in the experiments. None of these showed specific agglutination by anti-344 rabbit serum, by the convalescent sera of the donors, nor by a known positive convalescent human serum. Three of the donors, however, did show a rise in agglutinin titer to streptococcus No. 344 in samples of convalescent serum (Table I). From the volunteers, 26 additional strains of indifferent streptococci were isolated. Nineteen of these when tested showed non-specific agglutination reactions with rabbit immune and human convalescent serum. All samples of serum obtained from the 15 volunteers and the 16 men in the non-inoculated group were tested against No. 344 and the 19 strains isolated from the recipients. None of these samples of serum showed a rise in agglutinin titer to any of the strains. This evidence indicates that the indifferent streptococcus probably did not play a significant etiological rôle in the illnesses developing in any of the volunteers.

It has been suggested (5) that "the development of cold agglutinins may serve as a criterion for segregating some of the prevalent cases of primary atypical pneumonia until definite etiological agents are established." The inocula used in these experiments were obtained from 7 patients with primary atypical pneumonia, 5 of whom were shown to possess high titers of cold agglutinins in their sera. Three of the volunteers who received the inocula developed significant titers of cold agglutinins approximately 1 week following the onset of the febrile period. It has been found in this laboratory (4) that cold agglutinins first appear in the sera of some pa- tients with atypical pneumonia at approximately this time. Furthermore, the incidence of positive reactors in this study ( 25 per cent) compares favorably with what has been found in patients at Fort Bragg. Approximately 35 per cent of patients with atypical pneumonia may show the phenomenon at some stage of illness. It seems probable from the foregoing observations, therefore, that the clinical illnesses in the 3 inoculated volunteers who developed cold agglutinins were closely related, to those in the patients originally studied at Fort Bragg and from whom the inocula were obtained.

\section{SUMMARY}

A preliminary experiment has been conducted in human volunteers in an attempt to transmit primary atypical pneumonia. Unfiltered throat washings and sputa obtained from patients early in the course of the characteristic disease were inoculated into the respiratory tract of 12 individuals. The volunteers were kept in groupisolation for a period of 6 weeks and observed daily for the development of any signs of respiratory illness.

Respiratory illnesses developed in 10 of the 12 volunteers. These illnesses varied considerably not only in severity, but also in clinical manifestations, one from the other. They differed sharply from infections observed in a similar group of non-inoculated individuals.

Evidence is presented to indicate that illnesses closely resembling primary atypical pneumonia developed in certain of those who received the inoculations.

The members of the Commission wish to express their gratitude to the administrative staffs of Selective Service, the National Service Board for Religious Objectors, and the American Friends Service Committee for the cooperation which has made these studies possible. Mr. E. Gordon Alderfer of N.S.B.R.O. materially assisted this investigation from its inception. Dr. Alex M. Burgess, Jr., Medical Director, American Friends Service Committee, gave his complete support to the study. Without the continued enthusiastic cooperation of Dr. John $\mathbf{H}$. Ferguson, Director of Civilian Public Service Camp No. 108, and his administrative and medical staffs, this investigation would have been considerably restricted. Special thanks are due Major J. Ross Eakin, Superintendent of the Great Smoky National Park, for suspending the forestry project-work of the volunteers during the conduct of the experiment. 
Lieutenant Colonel Harold O. Brown, M.C., and Captain J. S. Raper, M.C., of the X-ray Department, Regional Hospital, Fort Bragg, rendered invaluable assistance in the interpretation of roentgenographic films. T/4 Morton G. Reifer, x-ray technician, and Mr. Walter A. Mickle, Jr., of the laboratory staff, assisted the Commission during the entire period of this investigation.

The Commission takes pleasure in acknowledging the assistance of the following members of the technical staff of the laboratory: Miss Barbara A. Mulliken, Thomas J. Oliver, 1st Lieutenant, Sn. C., Ralph L. Robinson, 1st Lieutenant, Sn. C., and Miss Irene A. Salamandra.

\section{BIBLIOGRAPHY}

1a. Gallagher, J. R., Bronchopneumonia in adolescence. Yale J. Biol. and Med., 1934, 7, 23.

b. Murray, M. E., Jr., Atypical bronchopneumonia of unknown etiology possibly due to a filterable virus. New England J. Med., 1940, 222, 565.

c. Daniels, 'W. B., Bronchopneumonia of unknown etiology in a girls' school. Am. J. M. Sc., 1942, 203, 263.

d. Longcope, W. T., Bronchopneumonia of unknown etiology (variety X). A report of thirty-two cases with two deaths. Bull. Johns Hopkins Hosp.; 1940, 67, 268.

e. Kneeland, Y., Jr., and Smetana, H. F., Current bronchopneumonia of unusual character and undetermined etiology. Bull. Johns Hopkins Hosp., 1940, 67, 229.

f. Dingle, J. H., and Finland, M., Virus pneumonias. II. Primary atypical pneumonias of unknown etiology. New England J. Med., 1942, 227, 378.

g. Dingle, J. H., Abernethy, T. J., Badger, G. F., Buddingh, G. J., Feller, A. E., Langmuir, A. D., Ruegsegger, J. M., and Wood, W. B., Jr., Primary atypical pneumonia, etiology unknown. Am. J. Hyg., 1944, 39, 67.

2a. Scadding, J. G., Disseminated focal pneumonia. Brit. M. J., 1937, 2, 956.

b. Ramsay, H., and Scadding, J. G., Benign bronchopulmonary inflammations associated with transient radiographic shadows. Quart. J. Med., 1939, 8, 79.

3a. Stokes, J., Kenney, A. S., and Shaw, D. R., A new filtrable agent associated with respiratory infections. Tr. and Stud., Coll. Physicians, Philadelphia, 1939, 6, 329.

b. Weir, J. M., and Horsfall, F. L., Jr., The recovery from patients with acute pneumonitis of a virus causing pneumonia in the mongoose. J. Exper. Med., 1940, 72, 595.

c. Eaton, M. D., Beck, M. D., and Pearson, H. E., A virus from cases of atypical pneumonia: relation to the viruses of meningopneumonitis and psittacosis. J. Exper. Med., 1941, 73, 641. d. Eaton, M. D., Meiklejohn, G., van Herick, W., and Talbot, J. C., An infectious agent from cases of atypical pneumonia apparently transmissible to cotton rats. Science, 1942, 96, 518.

4. Commission on Acute Respiratory Diseases, Cold hemagglutinins in primary atypical pneumonia and other respiratory infections. Am. J. M. Sc., 1944, 208, 742.

5. Peterson, O. L., Ham, T. H., and Finland, M., Cold agglutinins (autohemagglutinins) in primary atypical pneumonias. Science, 1943, 97, 167.

6. Thomas, L., Mirick, G. S., Curnen, E. C., Ziegler, J. E., Jr., and Horsfall, F. L., Jr., Serological reactions with an indifferent streptococcus in primary atypical pneumonia. Science, 1943, 98, 566.

7. Hirst, G. K., The quantitative determination of influenza virus and antibodies by means of red cell agglutination. J. Exper. Med., 1942, 75, 49.

8. Gallagher, J. R., Acute pneumonitis. A report of $\mathbf{8 7}$ cases among adolescents. Yale J. Biol. and Med., 1941, 13, 663.

9a. Reimann, H. A., and Havens, W. P., An epidemic disease of the respiratory tract. Arch. Int. Med., 1940, 65, 138.

b. Kornblum, K., and Reimann, H. A., The roentgenological aspects of an epidemic of acute respiratory tract infection. Am. J. Roentgenol., 1940, 44, 333.

10a. Commission on Acute Respiratory Diseases, Primary atypical pneumonia. Am. J. Pub. Health, 1944, 34, 347.

b. Commission on Acute Respiratory Diseases, Epidemiology of atypical pneumonia and acute respiratory disease at Fort Bragg, North Carolina. Am. J. Pub. Health, 1944, 34, 335.

11. Dochez, A. R., Shibley, G. S., and Mills, K. C., Studies in the common cold. IV. Experimental transmission of the common cold to anthropoid apes and human beings by means of a filtrable agent. J. Exper. Med., 1930, 52, 701.

12. Long, P. H., Doull, J. A., Bourn, J. M., and McComb, E., The etiology of acute upper respiratory infection (common cold). J. Exper. Med., 1931, 53, 447.

13. Stuart-Harris, C. H., Andrewes, C. H., Smith, W., Chalmers, D. K. M., Cowen, E. G. H., and Hughes, D. L., A Study of Epidemic Influenza: with Special Reference to the 1936-7 Epidemic. Medical Research Council, Special Report Series, No. 228. H. M. Stationery Office, London, 1938.

14. Burnet, F. M., and Clark, E., Influenza. A Survey of the Last 50 Years in the Light of Modern Work on the Virus of Epidemic Influenza. Macmillan and Company, Ltd., Melbourne, 1942.

15. Francis, T., Jr., Data reported at meeting of Am. Pub. Health Assn., New York, Oct. 1942. 